\section{Nutrição e promoção da saúde: perspectivas atuais}

\author{
Nutrition and health promotion: recent \\ perspectives
}

\footnotetext{
${ }^{1}$ Centro de Ciências da Saúde, Faculdades Federais Integradas de Diamantina, Diamantina, Brasil. 2 Escola Nacional de Saúde Pública Sergio Arouca, Fundação Oswaldo Cruz, Rio de Janeiro, Brasil.

Correspondência V. A. Ferreira

Departamento de Nutrição, Centro de Ciências da Saúde, Faculdades Federais Integradas de Diamantina. Rua da Glória 187,

Diamantina, $M G$

39100-000, Brasil.

vanessa.nutr@ig.com.br
}

\section{Abstract}

In recent years, health promotion has been introduced as a promising strategy for the public health field. The current study is intended to link nutrition into the recent debate on health promotion, analyzing the role of nutritionists in this process. Given the complexity of the Brazilian nutritional situation due to the nutritional transition currently under way in the country, the health promotion proposal points to both new perspectives and challenges for eating and nutrition. New demands in academic training and the nutritional care model tend to foster a search for partnerships, innovation in eating practices, and nutritional education aimed at the achievement of nutritional citizenship.

Health Promotion; Food and Nutrition Education; Nutritional Transition; Nutrition
Vanessa A. Ferreira 1

Rosana Magalhães 2

\section{Introdução}

Inicialmente podemos dizer que os primeiros autores que fizeram referência ao conceito de promoção da saúde foram Winslow 1 e Sigerist 2 . Ambos relacionaram o termo promoção da saúde às condições de vida das populações. Mais adiante Leavell \& Clark ${ }^{3}$ se apropriaram do conceito ao formularem o modelo da história natural da doença. Segundo os autores, a medicina preventiva compreenderia três níveis de prevenção e a promoção da saúde estaria incluída no nível primário, relacionada à saúde e ao bemestar dos indivíduos. Na realidade, o discurso da medicina social no século XIX também manteve aproximação com a promoção da saúde, ao correlacionar saúde e condições de vida, através das obras de autores como Villermé, na França e Chadwick, na Inglaterra 4,5. Nesse sentido, o termo promoção da saúde não é recente. No entanto, somente nos últimos vinte anos é que a promoção da saúde reaparece enquanto estratégia promissora para o campo da Saúde Pública. A valorização do conceito de promoção da saúde na atualidade surge como resposta à crescente medicalização, à baixa eficácia dos serviços de saúde e aos altos custos do setor.

Nessa direção, o movimento de promoção da saúde emerge de forma mais vigorosa, a partir da década de 70 nos países desenvolvidos - Canadá, Estados Unidos e Europa Ocidental. É particularmente no Canadá que se observa a 
retomada do conceito de promoção da saúde, com a divulgação no ano de 1974, do Informe Lalonde (A New Perspective on the Health of $\mathrm{Ca}$ nadians). Esse documento colocou a promoção da saúde no patamar do planejamento estratégico. Tal proposta visava, sobretudo, a enfrentar os altos custos com a assistência médica no país. O Informe Lalonde obteve significativo impacto, propiciando o desdobramento do recente movimento de promoção da saúde em todo o mundo. Mais adiante, em 1978, a Organização Mundial da Saúde (OMS) realizou em Alma-Ata, a I Conferência Internacional sobre Cuidados Primários de Saúde. Esse evento repercutiu de forma expressiva os sistemas de saúde em todo o mundo, estabelecendo a meta Saúde para Todos no Ano 2000. Nessa conferência reafirmou-se enfaticamente um conceito amplo de saúde. Saúde como o estado de completo bem-estar físico, mental e social, e não apenas como ausência de doença. Essa reconceituação de saúde foi fundamental nesse processo porque serviu de alicerce para o atual movimento de promoção da saúde.

Posteriormente, o Ministério da Saúde do Canadá e a OMS passaram a estruturar conceitos e práticas sobre promoção da saúde, favorecendo a realização da I Conferência Internacional sobre Promoção da Saúde realizada em Ottawa, no Canadá, no ano de 1986. Desse encontro resultou a Carta de Ottawa, considerada marco referencial da promoção da saúde ${ }^{6}$. A conferência reuniu participantes de todo o mundo e teve por intuito compartilhar experiências no setor. Na Carta de Ottawa foram definidas cinco áreas prioritárias de ação em promoção da saúde: políticas públicas saudáveis, criação de ambientes saudáveis, reforço à ação comunitária, desenvolvimento de habilidades pessoais e reorientação dos serviços de saúde. Ainda nessa Carta, são descritos os pré-requisitos para a saúde: paz, educação, moradia, alimentação, renda, ecossistema saudável, justiça social e eqüidade. A promoção da saúde incorpora, portanto, uma noção ampliada de saúde e passa a se aproximar da idéia de qualidade de vida. Nessa abordagem, a saúde seria resultado de um amplo espectro associado com a qualidade de vida, compreendendo um conjunto de valores: justiça social, educação, renda, habitação, alimentação, nutrição, trabalho etc. Qualidade de vida por sua vez seria uma noção relacionada ao grau de satisfação dos indivíduos com o seu meio familiar, social e ambiental. Para Minayo et al. 7 (p. 8) "o termo abrange muitos significados, que refletem conhecimentos, experiências e valores de indivíduos e coletividades que a ele se reportam em variadas épocas, espaços e histórias diferentes, sendo, portanto uma construção social com a marca da relatividade cultural".
Mais adiante realizaram-se outras conferências internacionais, que propiciaram aprofundar as discussões em torno do tema da promoção da saúde.

Atualmente a promoção da saúde se apresenta como uma proposta inovadora para o setor, pois favorece a superação de impasses importantes no campo da Saúde Pública. Dentre eles, os desafios relacionados ao modelo biomédico dominante, reducionista, fragmentado, que não responde às demandas atuais de saúde. A promoção da saúde incorpora ainda, um conceito ampliado de saúde, que tem como objetivo propiciar um nível ótimo de vida e saúde às populações. Buss 4 (p. 165) sintetizou de forma apropriada a proposta de promoção da saúde ao afirmar: "A promoção da saúde, como vem sendo entendida nos últimos 20-25 anos, representa uma estratégia promissora para enfrentar os múltiplos problemas de saúde que afetam as populações humanas e seus entornos neste final de século (...) propõe a articulação de saberes técnicos e populares, e a mobilização de recursos institucionais e comunitários, públicos e privados, para seu enfrentamento e solução".

\section{A promoção da saúde no Brasil}

No Brasil, o movimento de promoção da saúde assume destaque no ano de 1986 com a realização da VIII Conferência Nacional de Saúde (CNS/MS). Em seu relatório final frisou-se: “ $O$ direito à saúde significa a garantia, pelo Estado, de condições dignas de vida e de acesso universal e igualitário às ações e serviços de promoção, proteção e recuperação da saúde, em todos os níveis, a todos os habitantes do território nacional, levando ao desenvolvimento pleno do ser humano em sua individualidade" 8 (p. 382). Em 1988, a Constituição Federal brasileira reforça o tema da promoção quando declara no seu art. 196: “ $A$ saúde é um direito de todos e dever do Estado, garantido mediante políticas sociais e econômicas que visem à redução do risco de doenças e outros agravos e ao acesso universal e igualitário às ações e serviços para sua promoção, proteção e recuperação". Na década de 90 o Sistema Único de Saúde (SUS), por meio da Lei $n^{\circ}$. 8080/90, incorpora a noção ampla de saúde dentro do contexto da promoção ${ }^{9}$. Seguem-se em 1992 os eventos Cúpula da Terra e Rio 90: Agenda 21, em que se discutiu o tema dos ambientes saudáveis. Em 1999, o Ministério da Saúde no Brasil realiza o I Fórum Nacional sobre Promoção da Saúde, em Brasília. O objetivo do fórum consistiu na divulgação das experiências e na criação de espaços para debate em promoção da saúde no país. Posteriormente, a realização da X Conferência Nacional de Saúde 
promoveu o debate acerca dos modelos de atenção voltados para a qualidade de vida.

Atualmente, o Ministério da Saúde no Brasil tem subsidiado ações de educação em saúde, vigilância e reorganização da rede de assistência básica, com a implementação do Programa Saúde da Família (PSF), Programa Agentes Comunitários de Saúde (PACS), Bolsa Família e Renda Mínima, Aleitamento Materno, Programa de Educação e Saúde através do exercício físico e do esporte, Programa de Humanização no Pré-Natal e Nascimento, entre outros 10. Essas ações têm favorecido a inclusão do tema da promoção na agenda política de saúde do país 10 . Recentemente realizou-se no Rio de Janeiro, em maio de 2005, o I Seminário Brasileiro de Efetividade da Promoção da Saúde, evento promovido pela Escola Nacional de Saúde Pública Sergio Arouca, Fundação Oswaldo Cruz, cujo propósito foi analisar o impacto das ações sobre promoção da saúde no Brasil. Desse encontro resultou as propostas de expansão das experiências em promoção no país, a construção de uma rede de promoção da saúde e a criação de um programa de evidência de efetividade em promoção da saúde no Brasil, reunindo a Oficina Regional Latino-Americana da União Internacional de Promoção da Saúde e Educação para a Saúde (UIPES/ORLA), Associação Brasileira de Pós-graduação em Saúde Coletiva (ABRASCO) e outras instituições nacionais 10.

Torna-se apropriado ressaltar, que o discurso da promoção da saúde se insere no contexto das limitações do modelo biomédico, que estrutura toda a organização do complexo médico-industrial. As críticas a esse modelo se dirigem à hegemonia do enfoque curativo, reducionista, hospitalocêntrico e de alto custo. Tal proposta não equaciona os agravos decorrentes do perfil de morbimortalidade das sociedades contemporâneas 4 . Nesse sentido, a promoção da saúde favorece a superação desses impasses, porque amplia o conceito de saúde, incorpora novos princípios às práticas médicas, diferentes campos de conhecimento e, ainda, propõe estratégias organizacionais para os serviços de saúde. Dessa forma, a promoção da saúde visa a enfrentar os limites do modelo biomédico hegemônico e dos modelos de intervenção em saúde pública, apontando novos direcionamentos para o setor. Dentro desse contexto, acreditamos que a promoção da saúde situa-se em processo de construção teórica e prática. Aponta para o redirecionamento das políticas e programas de saúde no país. Assim sendo, o debate sobre promoção da saúde nos remete a mudanças nas ações tradicionais no campo da saúde pública e, conseqüentemente, elas incluem as práticas nutricionais. Este trabalho tem por objetivo contribuir para o debate em torno da promoção da saúde, a partir da aproximação do tema com o campo da alimentação e nutrição.

\section{O campo da alimentação e nutrição no debate da promoção da saúde}

A alimentação e nutrição constituem direitos humanos fundamentais consignados na Declaração Universal dos Direitos Humanos e são requisitos básicos para a promoção e a proteção da saúde, possibilitando a afirmação plena do potencial de crescimento e desenvolvimento humano com qualidade de vida e cidadania. $\mathrm{O}$ direito à alimentação é um direito do cidadão, portanto, dever do Estado e responsabilidade da sociedade. Nesse sentido, a busca em garantir o direito à alimentação de qualidade para todos os indivíduos, passa pela construção de um novo paradigma de sociedade, que tenha como eixo central a qualidade de vida do ser humano 11.

Nessa direção, a Declaração de Adelaide 12, documento produzido na II Conferência Internacional de Promoção da Saúde realizada em 1988 na Austrália, destacou como uma das áreas prioritárias para a promoção da saúde: a alimentação e nutrição. A eliminação da fome, da má nutrição e dos agravos relacionados ao excesso de peso, foi considerada meta essencial para a melhoria da qualidade de vida das coletividades. De acordo com essa declaração, as ações no setor de alimentação devem se estruturar no contexto da segurança alimentar e nutricional, para a garantia do acesso universal à ração de qualidade em quantidade suficiente, com respeito aos aspectos sócio-culturais das populações. Para tal, são propostas medidas de integração entre os setores de produção, distribuição e acesso aos alimentos, a fim de assegurar o direito eqüitativo e justo à alimentação 11. Nesse mesmo ano, a Constituição Federal brasileira reconhece a alimentação como um direito humano universal. Mas adiante, no ano de 1999, o Ministério da Saúde no Brasil elabora a Política Nacional de Alimentação e Nutrição, reforçando a questão da garantia à ração alimentar digna 13 .

No Brasil, inúmeros são os desafios encontrados para que a população alcance um nível ótimo de nutrição. A complexidade dos problemas alimentares, advindos da transição nutricional em curso no país, tem imposto reformulações urgentes ao setor, a fim de responder as novas demandas alimentares. A transição nutricional pode ser conceituada como um fenômeno no qual ocorrem mudanças nos padrões de distribuição dos problemas alimentares de uma população. Em geral, refere-se à passagem da desnutrição 
para a obesidade ${ }^{14}$. No Brasil, a transição nutricional assumiu um perfil singular 14,15. Assim, sem equacionar satisfatoriamente os problemas alimentares relacionados à carência absoluta de alimentos, o país convive com perfis nutricionais distintos, por vezes, sobrepostos. Nota-se a evolução da desnutrição, a permanência das anemias carenciais e o incremento da obesidade e dos agravos relacionados a ela. Tal perfil tem exigido dos profissionais nutricionistas uma reflexão mais aprofundada de seu papel enquanto agente ativo nesse processo. Sem dúvida, a superação dos problemas alimentares no país envolve aspectos intricados. Nesse contexto, a proposta de promoção da saúde sugere um caminho promissor para o campo da alimentação e nutrição. Fundamentalmente por se tratar de uma estratégia que articula diferentes setores e atores sociais, onde perpassam questões como: modelo de saúde sob o enfoque da integralidade; articulação de saberes técnicos e populares; capacitação dos indivíduos; parcerias nas ações; intersetorialidade de órgãos públicos e privados; reforço à ação comunitária; educação popular; cidadania; ética pública; entre outros. Qual seria então o papel da nutrição frente à proposta de promoção da saúde? O que esse debate poderia apontar de novo para as práticas nutricionais? Quais as implicações desse modelo para o profissional nutricionista?

\section{Nutrição para a promoção da saúde}

Tomando como referência os campos de ação em promoção da saúde supracitados, a proposta deste trabalho é refletir sobre a atuação do profissional nutricionista no contexto da promoção da saúde. Nessa direção, no que compreende a adoção do modelo de atenção sob o enfoque da integralidade, o desafio imposto à categoria dos nutricionistas remete a mudanças estruturais, que têm início na própria formação acadêmica do profissional. Analisando os currículos dos cursos de nutrição, notamos que eles se apresentam moldados sob a vertente biológica da atenção clínico-assistencial estruturada no modelo biomédico dominante. E, em contrapartida, na deficiente abordagem prestada às questões políticas, sócio-econômicas e culturais necessárias, não somente para a construção de uma análise crítica a respeito da conjuntura alimentar dos grupos populacionais, mas para as medidas de atenção em saúde. A repercussão desse fato está na dificuldade de articular os conhecimentos biológicos da nutrição com sua dimensão social, prejudicando a percepção da totalidade do fenômeno sob estudo 16,17 . Na prática, essas implica- ções se apresentam sob a difícil tarefa encontrada pelo nutricionista em realizar uma análise mais aprofundada acerca da dinâmica dos problemas alimentares 17. Conseqüentemente, esse fato gera entraves na implementação de medidas de intervenção mais consistentes. Vale ressaltar que essa tem sido uma tendência das profissões da área da saúde. A formação tecnicista se apresenta como um dos principais empecilhos para a prática da integralidade. De tal forma que, atualmente, emerge o movimento para a reforma na graduação. Nesse sentido, há urgência na definição de uma política de Estado para a formação de profissionais de saúde, com base no modelo da integralidade 18,19,20. Ainda sob esse aspecto, não tem sido incomum verificar a insatisfação dos profissionais nutricionistas com a sua prática, deflagrada através de sentimentos de frustração e impotência frente ao seu trabalho cotidiano, como têm demonstrado alguns estudos nacionais com esta categoria 21,22,23. A inclusão do campo da alimentação e nutrição no debate da promoção da saúde parece apontar para avanços importantes, abrindo novos horizontes para a atuação desses profissionais, os nutricionistas sociais. Se por um lado temos a possibilidade de repensar nossa prática enquanto profissionais de saúde e nossa atuação no campo da saúde pública, de outro temos importantes percalços a serem superados. O desafio, portanto, está em superar práticas convencionais e fragmentadas, decorrentes desse modelo de formação profissional.

A ação que envolve o segundo campo de atuação em promoção da saúde refere-se à articulação de saberes técnicos e populares. Essa ação se aproxima fundamentalmente da discussão anterior, pois implica transcender modelos tradicionais de atuação em nutrição. Ações de promoção da saúde impõem a criação de espaços democráticos e participativos, a fim de estabelecer uma aproximação com a realidade dos indivíduos e das populações. O objetivo é tentar responder melhor às demandas de saúde e nutrição. Dessa forma, abordagens persuasivas e ações verticais devem ser substituídas por estratégias mais amplas provenientes da relação profissional-paciente, a partir do somatório de saberes técnicos e populares. Nesse aspecto, torna-se imprescindível superar a relação hierárquica entre o profissional e o usuário.

No âmbito das ações direcionadas à capacitação dos indivíduos, o propósito será garantir condições para que a população possa exercer sua autonomia decisória, optando por escolhas alimentares mais saudáveis. Nesse aspecto, a educação nutricional assume um papel fundamental para o exercício e fortalecimento da cidadania alimentar. A prática do nutricionista assume o 
desafio de promover uma educação nutricional eficaz, com ações que promovam mudanças nos hábitos alimentares dos indivíduos e de suas famílias. Sob esse aspecto é interessante destacar que na primeira publicação da OMS, que tratou especificamente da questão da educação nutricional, já se fazia referência sobre a importância do ensino de bons hábitos alimentares para as populações 24 . Assim, outro ponto fundamental a ser destacado quando se propõe a articulação do campo da alimentação e nutrição no contexto de promoção da saúde é justamente o tema da educação nutricional. Destacamos que a educação nutricional a qual nos referimos, diz respeito a um processo de aprendizagem e não de adestramento. Educar no sentido de um processo amplo de desenvolvimento da pessoa, na busca de sua integração e harmonização, nos diversos níveis do físico, do emocional e do intelectual 25. Deve envolver metodologia de ensino-aprendizagem para o desenvolvimento de habilidades individuais, que permitam enfrentar as questões relacionadas à alimentação e nutrição.

A análise acerca da educação nutricional no Brasil 26 revela desafios importantes à categoria profissional dos nutricionistas. Embora seja disciplina obrigatória dos currículos de nutrição e conteúdo das práticas desenvolvidas por esses profissionais, a fragilidade de integrá-la com disciplinas de cunho social tal como nos referimos anteriormente, tende a comprometer a qualidade e a eficácia dos programas de educação nutricional. Isso porque a contextualização dos problemas alimentares, a partir da agregação de disciplinas provenientes do campo das ciências humanas e sociais, mostra-se essencial nesse processo ${ }^{17}$. Observa-se que o modelo tradicional da educação nutricional normalmente não propõe métodos de ensino problematizadores e construtivistas. Em geral, o método tradicional de educação nutricional se concentra nos efeitos biológicos dos nutrientes no organismo, na orientação alimentar, e ainda, na preservação das propriedades nutritivas dos alimentos envolvendo as técnicas de preparo e armazenamento. Tais ações podem ser consideradas benéficas já que, em algumas circunstâncias, verifica-se que os indivíduos apresentam desconhecimento sobre a questão alimentar. Entretanto, é preciso reconhecer que o Brasil é um país extenso, que apresenta contrastes e desigualdades sociais que acabam por comprometer o acesso da população ao direito universal à alimentação. A desigualdade no acesso à ração alimentar é uma realidade vivenciada por parcelas significativas da população brasileira. Ou seja, o país apresenta uma enorme fragilidade no que se refere à questão da segurança alimentar e nutricional 27. Assim, nas sociedades onde existem agravos relacionados à má nutrição tal como o Brasil, verifica-se também desigualdades no acesso e na produção dos alimentos 27 . Nesse aspecto, medidas de democratização dos recursos produtivos por meio da inclusão dos indivíduos e das comunidades nesse processo são fundamentais. As ações de incentivo à agricultura familiar, a hortas comunitárias, a cooperativas agrícolas, se apresentam como alternativas viáveis. Devemos destacar que as desigualdades no acesso à ração alimentar digna, se refere a uma produção social e econômica, que deve ser combatida pelos nutricionistas sociais. Torna-se necessário ressaltar ainda, a importância do diagnóstico consistente por parte dos nutricionistas, a fim de reconhecer as reais condições de nutrição dos indivíduos e comunidades assistidas. A partir dele será possível propor medidas educativas de maior resolutividade. Dessa maneira, se os problemas alimentares decorrem da dificuldade de acesso aos alimentos, pouco impacto terão as técnicas de aconselhamento e orientação alimentar.

Ações que se propõem ao reforço à ação comunitária devem sobrepor atividades de educação nutricional de aconselhamento. Demandam a criação de espaços públicos, que possam desenvolver o pensamento crítico para a identificação de problemas e a elaboração de estratégias para sua superação. Implica a valorização de espaços para o debate e o estabelecimento de parcerias entre profissional-usuário-comunidade. No âmbito coletivo, ao educador caberia assumir seu papel de agente político. A participação na formulação das políticas, programas e ações em alimentação e nutrição junto ao poder público, assim como, sua articulação com as organizações não-governamentais, o setor privado e a sociedade civil são fundamentais. A inserção nos espaços educativos, tais como creches, escolas e nos meios de comunicação de massa também devem ser fomentadas. É imprescindível ainda, que o educador promova, ou esteja engajado, em debates, encontros, seminários, sejam eles locais ou nacionais, para o fortalecimento das ações de educação nutricional.

A educação popular, por sua vez, compreende todos os aspectos já discutidos. Deve envolver atividades direcionadas a: mudança comportamental, informações sobre políticas públicas vigentes, assim como o incentivo ao exercício permanente dos diretos e deveres, especialmente os relacionados à alimentação. Importante enfocar nessas ações, informações nutricionais e educação com enfoque na higiene e saúde. Por fim, o último campo de ação em promoção da saúde remonta à questão da intersetorialidade das ações $\mathrm{e}$ será discutido a seguir. 


\section{O desafio da intersetorialidade}

O debate em torno da promoção da saúde parte do pressuposto de que saúde e qualidade de vida não dependem do oferecimento isolado de um dado serviço, mas sim, da oferta de um conjunto de condições de vida e de trabalho 28 . Trata-se, portanto, de uma proposta que implica formação de alianças, estabelecimento de parcerias e intersetorialidade nas ações. A questão da intersetorialidade apresenta-se como principal estratégia para implementação da proposta de promoção da saúde. Consiste no reconhecimento de que o setor saúde isoladamente não consegue responder às demandas de saúde da população, criando com isso a necessidade de articular as ações de saúde com os demais setores da sociedade. Buss \& Ramos 29 , ao fazerem referência ao tema da intersetorialidade, alertam que, para sua efetivação, há necessidade de superar e romper as barreiras comunicacionais. Para os autores são elas que impedem o diálogo entre os diferentes setores.

Refletindo especificamente sobre a prática do nutricionista, a questão intersetorial pode nortear a atuação destes profissionais nos diversos cenários, que ultrapassam o âmbito das unidades de saúde. Isso porque, tradicionalmente, a prática do nutricionista em unidades de atenção primária de saúde se configura pela atuação do profissional no consultório, por meio do atendimento ambulatorial, integrado ou não, ao trabalho em equipe. Cabe ao nutricionista promover intervenção alimentar através do atendimento individualizado ou em grupo, e realizar atividade preventiva de educação nutricional. São ainda atribuições do profissional nutricionista nas unidades locais: a vigilância nutricional e a elaboração de estatísticas sobre as atividades desempenhadas nas unidades. Assim, o cotidiano de práticas desempenhadas por esses profissionais nas unidades básicas de saúde apresenta-se circunscrito ao consultório, ou no máximo, em atividades dentro da própria unidade. A partir da intersetorialidade é possível inserir a nutrição em outros ambientes sociais, tais como nas escolas públicas e privadas; na comunidade por intermédio dos agentes comunitários de saúde, das associações de moradores e do próprio PSF proposto pelo Ministério da Saúde; no ambiente de trabalho formal; nas ações de vigilância sanitária; nos órgãos de comunicação em geral; nas organizações não-governamentais (ONGs); em zonas agrícolas; enfim, inúmeros são os espaços nos quais a nutrição pode estar articulada. Fundamentalmente, essa inserção coloca a temática da nutrição no cerne das discussões nos diversos espaços sociais, fortalecendo dessa maneira, esse campo de práticas.
Importante destacar que a estratégia da intersetorialidade é especialmente importante para a área de segurança alimentar e nutricional. Reconhecidamente a questão alimentar é complexa e impõe a formação de alianças por envolver diferentes esferas das políticas públicas, tais como a produção, comercialização, distribuição e consumo de alimentos, controle de qualidade, estímulo à educação nutricional, dentre outras ações ${ }^{30}$. A intersetorialidade se apresenta, assim, como uma estratégia prioritária na construção de espaços legítimos para o diálogo entre os diferentes setores envolvidos na dinâmica alimentar, visando a assegurar o acesso à alimentação digna.

O estabelecimento de parcerias impõe desprendimento, ousadia, envolvimento e criatividade para os profissionais nutricionistas porque rompe com as formas convencionais de atuação da categoria. Nesse sentido, parcerias setoriais, o somatório de saberes técnicos e populares, a superação do modelo biomédico dominante com a incorporação da visão integral dos indivíduos, assim como, a habilidade técnica desses profissionais (com ênfase na educação nutricional), nos parece ser o caminho para incorporar a nutrição dentro da proposta de promoção da saúde. Sabemos não se tratar de tarefa fácil, porém ela nos é requisitada e, portanto, necessária. Como mencionamos anteriormente, a transição nutricional em curso no país, justifica a mobilização dos nutricionistas sociais nas ações de promoção da saúde.

\section{Conclusão}

Por fim, destacamos que os desafios impostos para o campo da nutrição no contexto da promoção da saúde são inúmeros. No entanto, tais desafios apontam para a possibilidade de compatibilizar diferentes olhares e perspectivas teórico-metodológicas na direção do diálogo interdisciplinar. Repensando seu lugar como profissional da área da saúde e reintegrando as práticas alimentares ao conjunto de experiências, saberes e intervenções voltadas ao atendimento das necessidades sociais dos indivíduos e grupos sociais, o nutricionista pode avançar na construção de uma proposta de atuação capaz de contribuir para a redução das iniqüidades em saúde e a garantia da qualidade de vida. Nesse esforço, portanto, a discussão da efetividade das ações, o aprendizado com as experiências em curso, a escolha de novas ferramentas metodológicas devem penetrar na formação e no exercício profissional, tendo por intuito romper a fragmentação tecnicista tradicional e promover 
a integralidade, a intersetorialidade, a eqüidade e a participação social. A mobilização em torno de uma nova prática para o nutricionista esta- belece, assim, elos fortes com o debate acerca da formulação e implementação de políticas e ações de promoção da saúde.

\section{Resumo}

A promoção da saúde tem ao longo dos últimos anos se apresentado como uma estratégia promissora para o campo da Saúde Pública. Este estudo propõe articular o tema da nutrição no debate contemporâneo da promoção da saúde, analisando o papel dos nutricionistas neste processo. Frente ao reconhecimento da complexidade do quadro alimentar brasileiro advindo da transição nutricional em curso no país, a proposta de promoção da saúde aponta perspectivas, mas também desafios ao campo da alimentação e nutrição. Em linhas gerais, novas exigências relacionadas à formação acadêmica e ao modelo de atenção tendem a fortalecer a busca de parcerias, inovação das práticas alimentares à educação nutricional voltada à construção da cidadania alimentar.

Promoção da Saúde; Educação Alimentar e Nutricional; Transição Nutricional; Nutrição

\section{Colaboradores}

V. A. Ferreira foi responsável pela elaboração, redação e revisão do artigo. R. Magalhães participou da elaboração e revisão do artigo. 


\section{Referências}

1. Winslow CEA. The untilled fields of public health. Science 1920; 51:23.

2. Sigerist H, editor. The university at the crossroad. Nova York: Henry Schumann Publisher; 1946.

3. Leavell S, Clark EG. Medicina preventiva. São Paulo: McGraw-Hill; 1976.

4. Buss PM. Promoção da saúde e qualidade de vida Ciênc Saúde Coletiva 2000; 5:163-77.

5. Czeresnia D, Freitas CM, organizadores. Promoção da saúde: conceitos, reflexões, tendências. Rio de Janeiro: Editora Fiocruz; 2003.

6. Buss PM. Uma introdução ao conceito de promoção da saúde. In: Czeresnia D, Freitas CM, organizadores. Promoção da saúde: conceitos, reflexões, tendências. Rio de Janeiro: Editora Fiocruz; 2003. p. 15-38.

7. Minayo MCM, Hartz ZMA, Buss PM. Qualidade de vida e saúde: um debate necessário. Ciênc Saúde Coletiva 2000; 5:7-18.

8. Ministério da Saúde. 8a Conferência Nacional de Saúde: relatório final. Brasília: Ministério da Saúde; 1986.

9. Ministério da Saúde. Lei Federal no . 8.080, de 19 de setembro de 1990. Dispõe sobre as condições para a promoção, proteção e recuperação da saúde, a organização e o funcionamento dos serviços correspondentes e dá outras providências. Diário Oficial da União 1990; 19 set.

10. Buss PM. Promoção da saúde no Brasil. I Seminário Brasileiro de Efetividade da Promoção da Saúde. http://www.ensp.fiocruz.br/eventos_novo/ dados/arq547.ppt (acessado em 20/Jul/2005).

11. Valente FLS. Direito humano à alimentação: desafios e conquistas. São Paulo: Editora Cortez; 2002.

12. Ministério da Saúde. As cartas da promoção da saúde. Brasília: Ministério da Saúde; 2002.

13. Ministério da Saúde. Política nacional de alimentação e nutrição. Brasília: Ministério da Saúde; 1999.

14. Kac G, Velásquez-Melendez G. A transição nutricional e a epidemiologia da obesidade na América Latina. Cad Saúde Pública 2003; 19 Suppl 1:S4-5.

15. Escoda MSQ. Para a crítica da transição nutricional. Ciênc Saúde Coletiva 2002; 7:219-26.

16. Sichieri R, Coitinho D, Pereira R, Marins V, Moura A Variações temporais do estado nutricional e do consumo alimentar no Brasil. Physis (Rio de J) 1997; 7:31-50.
17. Bosi MLM. A face oculta da nutrição: ciência e ideologia. Rio de Janeiro: Editora Espaço e Tempo; 1988.

18. Bosi MLM. Profissionalização e conhecimento: a nutrição em questão. São Paulo: Editora Hucitec; 1996.

19. Pinheiro R, Mattos RA, organizadores. Construção da integralidade: cotidiano, saberes e práticas em saúde. Rio de Janeiro: Eduerj/ABRASCO; 2003.

20. Ceccim RB, Feuerwerker LCM. Mudança na graduação das profissões de saúde sob o eixo da integralidade. Cad Saúde Pública 2004; 20:1400-10.

21. Mattos RA. A integralidade na prática (ou sobre a prática da integralidade). Cad Saúde Pública 2004; 20:1411-6.

22. Prado SD. Autonomia tentadora e reificada: estudo sobre nutricionistas dos Centros Municipais de Saúde do Rio de Janeiro [Dissertação de Mestrado]. Rio de Janeiro: Escola Nacional de Saúde Pública, Fundação Oswaldo Cruz; 1993.

23. Amorim STSP, Moreira H, Carraro TE. A formação de pediatras e nutricionistas: a dimensão humana. Rev Nutr 2001; 14:111-8.

24. Bosi MLM. Trabalho e subjetividade: cargas e sofrimentos na prática da nutrição social. Rev Nutr 2000; 13:107-15.

25. Ritchie JAS. Buenos hábitos en la alimentación: métodos para inculcarlos al público. Rome: Food and Agricultural Organization; 1951. (Estudios sobre Nutrición, 6).

26. Boog MCF. Educação nutricional: passado, presente e futuro. Rev Nutr 1997; 10:5-19.

27. L'Abbate S. As políticas de alimentação e nutrição no Brasil. II. A partir dos anos setenta. Rev Nutr $1989 ; 2: 7-54$

28. Valente FLS. Fome e desnutrição: determinantes sociais. São Paulo: Editora Cortez; 1988.

29. Buss PM, Ramos CL. Desenvolvimento local e agenda 21: desafios da cidadania. Cadernos da Oficina Social 2000; 3:13-65.

30. Inojosa RM, Junqueira LAP. O setor saúde e o desafio da intersetorialidade. Cad FUNDAP 1997; 21:156-64

Recebido em 01/Jun/2005

Versão final reapresentada em 04/Jan/2007

Aprovado em 10/Jan/2007 